\title{
EN LOS OJOS DE GULIVER: \\ INFORMACIÓN CONTEXTUAL Y SENTIDO DE ORIENTACIÓN ${ }^{\star}$
}

\author{
Rodrigo I. Mora ${ }^{\star}$
}

Escuela de Arquitectura, Faculta de Arquitectura, Arte y Diseño, FAAD, Universidad Diego Portales, Chile.

\begin{abstract}
RESUMEN
Este manuscrito estudia el sentido de orientación de las personas en situaciones en que existe información de tipo contextual, que permite a las personas a ver locaciones muy distantes. Para ello se preguntó a 133 individuos indicar la ubicación de cinco hitos próximos y lejanos de la ciudad de Valparaiso, en 2 situaciones distintas: miradores urbanos y lugares en el medio de la trama urbana en el que la visión de información contextual no es posible. Los resultados mostraron que no existía una asociación entre la Precisión Promedio por Persona y el sexo, edad, o el grado familiaridad, pero que sí con el lugar donde se habian hecho estas estimaciones.
\end{abstract}

Palabras Claves: orientación; información contextual; visión alocéntrica; Valparaíso.

\section{In GULliVER'S EYES: CONTEXTUAL INFORMATION AND SENSE OF ORIENTATION}

\begin{abstract}
This manuscript examines how people gain a sense of orientation in scenarios where large-scale, contextual information is available. Two locations of the city of Valparaiso where one can see the entire city from the heights were selected for an experiment, as well as two areas in the middle of the urban grid where no large-scale information was available. A total of 133 people were asked to point to five non-visible landmarks in these scenarios and their estimations were compared with the real position of each target. Results showed no association between this value and people's gender, age or familiarity with the neighborhood. However, a positive relationship was found between this value and the places in which estimates were made.
\end{abstract}

Keywords: orientation; contextual information; vision allocentric; Valparaíso.

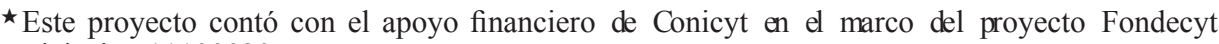
Iniciación 11100020

$\star \star$ Dirección: Avenida España 1680, Santiago, Chile. Correo electrónico: rodrigo.mora@udp.cl
} 


\section{1.- INTRODUCCIÓN}

Ser capaz de orientarse en el medio construido es fundamental para la especie humana. Gracias a ello, las personas pueden alcanzar un destino en un medio ambiente desconocido, inventar una nueva ruta cuando la acostumbrada está bloqueada o dar a un tercero indicaciones sobre cómo llegar a un lugar específico. En la mayoría de los casos, estas actividades se llevan a cabo sin mayor problema, pero en algunas circunstancias, ya sea porque la propia arquitectura del medio construido es confusa o por la ausencia de señalización (o el mal diseño de ésta), las personas son incapaces de orientarse. Como resultado de lo anterior, extravían temporalmente el rumbo o simplemente no logran llegar a sus destinaciones, se pierden. Los sentimientos más comunes que siguen a esto son la angustia, la culpa y un sentimiento de inseguridad e impotencia.

Se ha dicho que para ser capaces de orientarse, las personas deben primero formarse una imagen mental del mundo (GOLLEDGE et al., 1985). Esta representación se conoce, en la terminología usada por la cognición espacial, como "mapa cognitivo" (TOLMAN, 1948), survey map (SIEGEL; WHITE, 1975), o "representación configuracional del espacio" (DOWNS; STEA, 1973). Todos estos términos se refieren a una visión abstracta y aproximada del medio ambiente, cuya característica última es la de dar cuenta de cómo distintos elementos constituyentes del mundo se relacionan entre sí. Son, en síntesis, representaciones de tipo topológicas o configuracionales, que muestran al mundo como si fuera visto "por un pájaro" (THORNDYKE; HAYES-ROTH, 1982).

El factor más frecuentemente citado como interviniente para el desarrollo de un mapa cognitivo es el grado de exposición (o familiaridad) al medio. Varios autores han señalado (APPLEYARD, 1970; FOLEY; COHEN, 1984; GOLLEDGE, 1992; MONTELLO, 1992) que a mayor exposición con el medio, más refinado (métricamente y configuracionalmente), se torna la imagen mental de una persona. Menos consenso existe, sin embargo, sobre si existen diferencias en las capacidades de orientarse en hombres y mujeres: mientras la mayoría de los estudios no acusan mayores diferencias (KITCHIN, 1996), algunos reportan un rendimiento levemente mejor en hombres (WARD; NEWCOMBE; OVERTON, 1986; MONTELLO et al., 1999). La edad de las personas, por otro lado, sido señalada como una variable interviniente de menor escala en la orientación (KIRASIC, 2000).

No obstante, varios autores han señalado que es fundamentalmente gracias a la visión que la mayoría de las personas forman su mapa cognitivo (MONTELLO, 1992), pues este sentido les permite percibir, identificar y reconocer elementos del medio ambiente (sean éstos hitos, lugares o la estructura de una ciudad o el sistema de circulaciones de un edificio). Con el tiempo, esta representación se refinaría, ganando precisión métrica y configuracioonal (THORNDYKE; HAYES-ROTH, 1982; GOLLEDGE; STIMSON, 1997), lo que a su vez permitiría a las personas realizar evaluaciones más precisas de la distancia entre distintos lugares. 


\section{2. - EL ROL DE LA INFORMACIÓN CONTEXTUAL EN LA ORIENTACIÓN}

La literatura sobre cognición espacial ha sido pródiga en estudios sobre orientación. Al respecto, la técnica más común para dilucidar cuán orientadas son las personas ha sido el pedirles que indiquen con su mano o usando elementos creados para tal efecto, la ubicación y la distancia (en metros o minutos), de ciertos lugares o hitos. En los últimos treinta años se han realizado estudios de orientación tanto en medios reales como campus universitarios (MONTELLO; PICK, 1993; SAUCIER et al., 2002), o laberintos creados especialmente para tal efecto (SCHMITZ, 1997), como en medios simulados en los cuales las personas deben ver una película (O'LAUGHLIN; BRUBAKER, 1998), un video (MOFFAT; HAMPSON; HATZIPANTELIS, 1998; WALLER; BEALL; LOOMIS, 2004) o una serie de diapositivas (HOLDING, C.; HOLDING, D., 1989). Por su parte, los métodos para recabar información sobre orientación han variado van desde aquellos que solicitan a las personas dibujar mapas (WARD; NEWCOMBE; OVERTON, 1986; COLUCCIA; MARTELLO, 2004), aquellos que solicitan descripciones verbales del medio (DENIS et al., 1999), o aquellos que usan aparatos tecnológicos modernos como Ipads (CARLSON et al., 2010), para señalar la dirección de uno u otro hito.

Varios son los resultados comunes de estos estudios. Se ha argumentado las personas ocupan distintas estrategias al momento de orientarse y "navegar" en el medio construido dependiendo del grado de familiaridad con el medio ambiente en que se mueven (LAWTON, 1996; HÖLSHER et al., 2006). Asimismo, se ha afirmado que la capacidad de orientación, medida a través de estimaciones de dirección, es afectada por factores como la orientación de las calles con respecto a la posición del observador (MONTELLO, 1991), o incluso por la existencia de "ejes naturales del cuerpo" (body axes) (TVERSKY, 2003), lo que daría como resultado el hecho de que las personas "acercaran" las estimaciones a estos ejes (KLIPPEL; MONTELLO, 2007). Por último, se ha afirmado que la orientación es "refinada", es decir, gana precisión y complejidad, a medida que las personas se familiarizan con el medio ambiente (FOLEY; COHEN, 1984; GOLLEDGE et al., 1985). Quizás más importante es el hecho de que la mayoría de estudios sobre la materia sugieren que lo clave para que las personas logren orientarse en el espacio es que puedan generar un "marco de referencia" que aglutine y ordene a los distintos lugares e hitos del espacio en un todo único (THORNDYKE; HAYES-ROTH, 1982; GÄRLING et al., 1984). Visto así, parece lógico pensar que la disponibilidad de información de gran escala sobre la ciudad, como la que se encuentra en miradores o en espacios con alta visibilidad que permiten ver la ciudad desde las alturas, ayudaría a las personas a orientarse.

Estudios en la materia parecen apuntar en esta dirección. Se ha sugerido que las personas usaban información de tipo "contextual" (fundamentalmente elementos geográficos de gran escala) para establecer la ubicación relativa de elementos y lugares en el medio construido (ALLEN; SIEGEL; ROSINSKI, 1978). Steck y Mallot (2000), por otro lado, demostraron que las personas usan alternativamente hitos de menor y mayor escala al moverse en el medio ambiente, pues mientras los primeros permiten monitorear el desarrollo de una trayectoria en particular, los se- 
gundos permitirían conservar las direcciones generales trazadas para ir de un lugar a otro. Finalmente, Gärling (GÄRLING; LINDBERG; MANTYLA, 1983) mostró que el restringir artificialmente la disponibilidad de información contextual, a través de la restricción de visión perimetral, hacía más difícil a las personas establecer la ubicación de hitos en el espacio y navegar en el medio construido.

Sin embargo, ninguno de estos estudios se ha realizado en situaciones más ecológicamente válidas (o más reales), como es el caso de ciudades con geografías abruptas con miradores naturales. Asimismo, la mayoría de los estudios se han realizado en situaciones en que las personas tienen una visión egocéntrica del mundo, es decir, tienen las perspectiva de un transeúnte que va por una calle que no puede observar las cosas desde arriba, como lo haría un pájaro. ¿Pero qué sucede en los casos en se tiene una visión de tipo alocéntrica, es decir, donde el espacio construido se ve en su totalidad y por lo tanto donde es posible reconocer hitos y lugares alejados entre sí y carentes de conexión visual?

Esta es la pregunta fundamental de este artículo. Acá se postula que la presencia de información contextual de tipo alocéntrica del entorno, facilita la localización de lugares e hitos no visibles por las personas, pues les provee un marco de referencia espacial desde donde el cual éstas pueden inferir la localización de lugares no visibles. Específicamente, acá se plantea que las estimaciones sobre la ubicación relativa de hitos y lugares, medida en grados desde un punto dado, son más precisas en lugares en los que se cuenta con información contextual de tipo alocéntrica, que en lugares que no proveen esta información.

\section{3.- MÉTodo}

\section{Escenarios}

El experimento tuvo lugar en la ciudad de Valparaiso, la tercera ciudad en términos de importancia de Chile. Localizada a casi 100 kilómetros de Santiago, la ciudad se asienta en un anfiteatro natural conformado por treinta y tres cerros que convergen a un espacio reducido y plano (el Plan), desde donde salen varios ascensores urbanos que se dirigen a los cerros.

Construidos a finales del siglo 19, estos ascensores generan una plataforma superior y una inferior. Mientras las primeras constituyen verdaderos miradores desde donde se puede ver gran parte de la bahía, los segundos son lugares mucho más segregados desde el punto de vista visual, pues al estar inmersos en el medio de la trama urbana no permiten a las personas ver el entorno geográfico.

La figura 1 muestra el plano de la ciudad de Valparaiso y los dos escenarios ocupados en este experimento. El primero de ellos corresponde al ascensor Espíritu Santo, localizado en el cerro Bellavista, en el área central de la ciudad. El segundo de ellos es el ascensor Polanco, ubicado en el cerro homónimo, que se encuentra en un sector no central de la ciudad. La figura 2 muestra la vista panorámica que se tiene de ambos escenarios. 


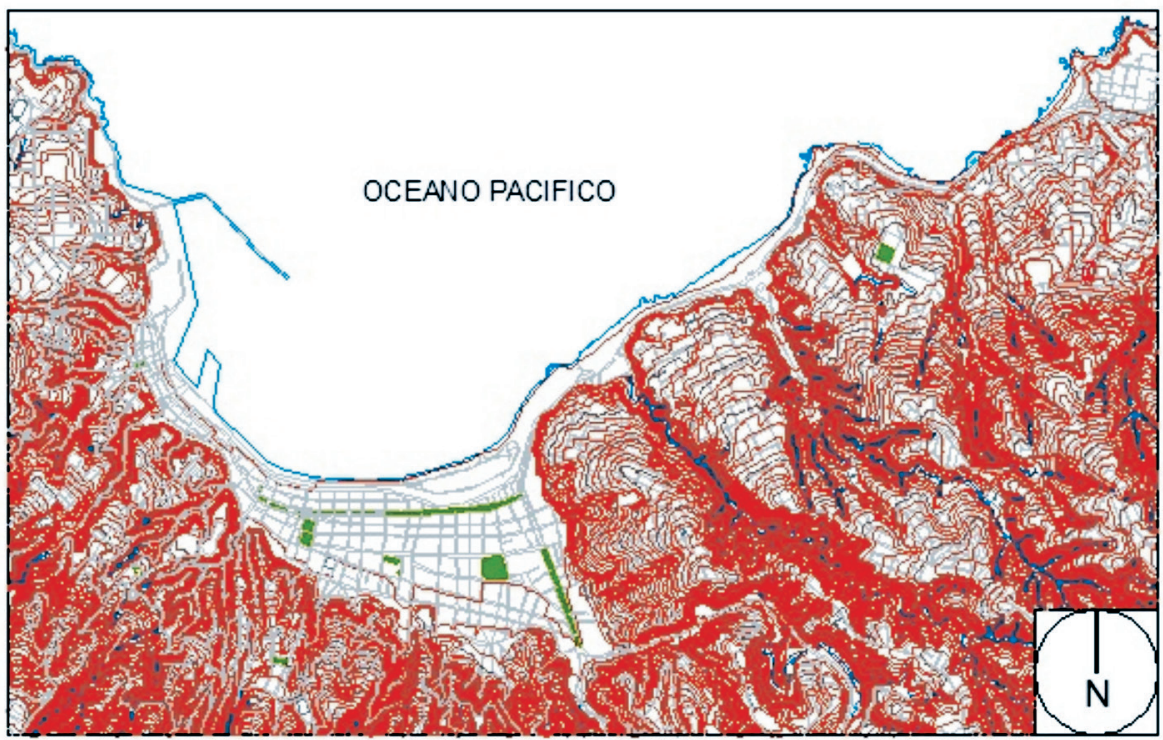

Figura 1: la bahía de Valparaíso

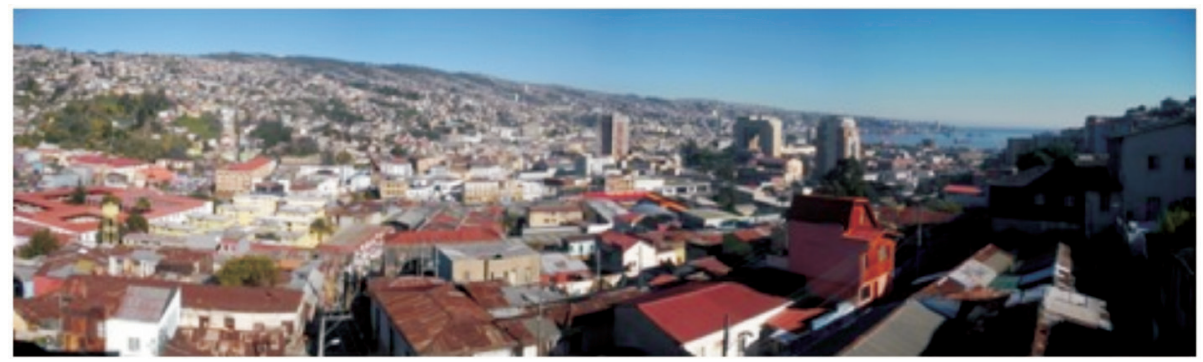

Figura 2: vista panorámica desde el ascensor Polanco.

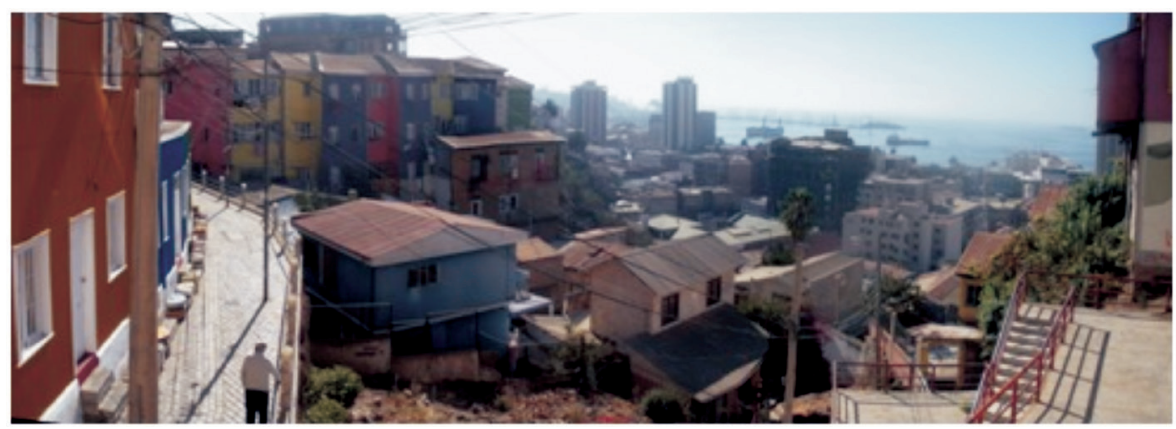

Figura 3: vista panorámica desde el ascensor Espíritu Santo. 


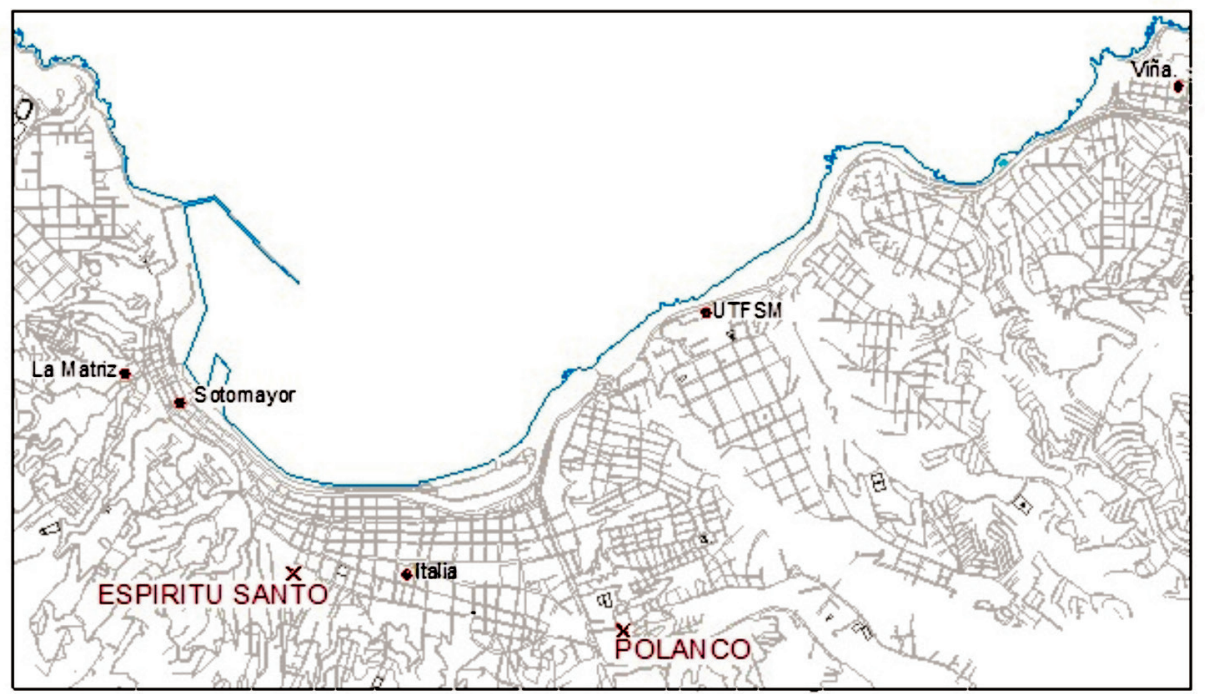

Figura 4: los escenarios elegidos en este experimento y las cinco hitos que debían ser apuntados.

\section{Materiales y Diseño}

En cada una de las cuatro situaciones definidas en este experimento (Plataforma Superior e inferior de ascensor Polanco, plataforma superior en inferior de ascensor Espíritu Santo), se encuestó a un grupo diferente de residentes. A éstos se les pidió localizar cinco hitos conocidos de la ciudad, que no eran visibles directamente ni en las plataformas superiores ni en las inferiores. Uno de estos hitos ni siquiera pertenecía a la ciudad de Valparaiso, sino a la vecina ciudad de Viña del Mar, que se encuentra conurbada a la primera.

Para hacer sus estimaciones, las personas debían ocupar un cartón circular de $6 \mathrm{~cm}$ de radio en cuyo centro había una aguja que rotaba. En el perímetro de este círculo estaban marcados los ángulos de 0 a 360. Al mover la aguja giratoria, los participantes podían definir la localización de cada uno de los hitos contemplados en el experimento. Las estimaciones hechas por las personas fueron luego comparadas con la posición real del hito según sus coordenadas geográficas, lo cual permitió establecer la primera de las variables dependientes del experimento: el Error Promedio por Persona (EPP), o distancia en grados desde la estimación hasta la posición real del hito. Se utilizó este procedimiento por ser una metodología ampliamente aceptada por la literatura sobre orientación espacial (TVERSKY, 1982; GOLLEDGE et al., 1993; KITCHIN, 1996). Adicionalmente, se definió una segunda variable dependiente ampliamente usada en estudios de cognición espacial: el tiempo de respuesta de los individuos en cada pregunta, esto es, desde que el encuestador preguntaba por la localización de cada hito hasta el minuto en que la estimación era realizada. Varios estudios (TVERSKY; BARATZ, 1985; GOLLEDGE, 1995) han mostrado que a mayor tiempo de respuesta menos es la precisión de las estimaciones de orientación. 


\section{Participantes y Procedimiento}

Un total de 133 sujetos (59 hombres y 74 mujeres, edad promedio 33 años, Desviación Estándar 13 años) participaron en este experimento. Setenta y uno de ellos fueron entrevistados en el ascensor Polanco, mientras sesenta y dos en el ascensor Espíritu Santo. La tabla 1 exhibe las características de los participantes de este experimento.

Las personas fueron abordadas en forma individual el momento de ir o venir de los ascensores. El entrevistador les preguntó se podían participar en un experimento sobre orientación en ciudades. Si aceptaban, se les llevaba a un lugar específico cercano a las plataformas superior o inferior del ascensor, donde se encontraba el instrumento de medición. Con el objeto de poder comparar las estimaciones hechas por las personas, se fijó en una posición específica el instrumento de medición.

Previo al testeo, las personas debieron familiarizarse con el instrumento de medición. Una vez que se sintieron cómodas con su uso, se les pidió apuntar a cada uno de los hitos mostrados en la figura 3. Los hitos a localizar tuvieron una secuencia aleatoria.

Al final de cada estimación, el entrevistador anotaba el tiempo que ésta duro y sus grados respecto del punto de origen $\left(0^{\circ}\right)$. Una vez terminada la serie de cinco estimaciones, las personas debían completar un pequeño cuestionario donde se les preguntaba su edad, tiempo de residencia, y autoevaluación sobre su sentido de orientación (bueno, regular, malo).

Tabla 1 - Información descriptiva de este experimento.

\begin{tabular}{lccccrr}
\hline & Hombres & Mujeres & $\begin{array}{c}\text { Plataforma } \\
\text { Superior } \\
\text { (mirador) }\end{array}$ & $\begin{array}{c}\text { Plataforma } \\
\text { Inferior } \\
\text { (nivel calle) }\end{array}$ & Total & Edad Promedio \\
\hline Polanco & $37(52.1 \%)$ & $34(47.9 \%)$ & $39(54.9 \%)$ & $32(45.1 \%)$ & 71 & $36(\mathrm{SD}=12.9)$ \\
\hline Espíritu Santo & $22(35.5 \%)$ & $40(64.5 \%)$ & $35(56.5 \%)$ & $27(43.5 \%)$ & 62 & $29(\mathrm{SD}=12.3)$ \\
\hline Total & $59(44.3 \%)$ & $74(55.7 \%)$ & $74(55.7 \%)$ & $59(44.3 \%)$ & 133 & $33(\mathrm{SD}=13)$ \\
\hline
\end{tabular}

Tabla 2 - Información descriptiva sobre tiempos de respuestas y errores por estimación.

\begin{tabular}{lrr}
\hline Ascensor & $\begin{array}{c}\text { Tiempo } \\
\text { Promedio por } \\
\text { Estimación } \\
\text { (en segundos) }\end{array}$ & $\begin{array}{c}\text { Error Promedio por } \\
\text { Estimación } \\
\text { (en grados) }\end{array}$ \\
\hline Polanco & $4.4(\mathrm{DE}=2.5)$ & $22.6(\mathrm{DE}=15.4)$ \\
\hline Espiritu Santo & $5(\mathrm{DE}=1.7)$ & $28.6(\mathrm{DE}=15.8)$ \\
\hline Total & $4.7(\mathrm{DE}=2.7)$ & $25.3(\mathrm{DE}=18)$ \\
\hline
\end{tabular}




\section{4.- Resultados}

\section{Auto-evaluación sobre sentido de orientación}

Las estimaciones hechas por las personas fueron agrupadas y analizadas estadísticamente mediante diferentes pruebas. Se usó pruebas de Chi-cuadrado para evaluar diferencias entre sexo, lugar de la estimación (superior o inferior), o entre cada plataforma. Los Análisis de Varianza (ANOVA) fueron usados para detectar diferencias entre grupos derivadas de la edad de las personas, su tiempo de residencia en cada cerro, o la autopercepción sobre sus capacidades de orientación.

Primero, no se encontraron diferencias entre la auto evaluación sobre el sentido de orientación en las plataformas superiores o inferiores de los ascensores (Chi-cuadrado 4.114, $\mathrm{p}>0.05$ ). Tampoco se encontró diferencias en la materia entre hombres y mujeres (Chi-cuadrado 4.215, $>>0.05$ ), ni entre los ascensores (Chi-cuadrado 2.87, $\mathrm{p}>0.05$ ). Asimismo, la edad de las personas no mostró una relación con la auto evaluación que hacían las personas sobre su capacidad de orientación (ANOVA (F: 1.81 p> 0.05), tal como lo mostró un análisis de varianza que dividió a los participantes en tres grupos (jóvenes adultos, de 18 a 30 años; adultos, de 31 a 50, y personas mayores, de más de 51 años). Finalmente, no se encontró diferencias estadísticamente significativas entre aquellos que habían vivido cinco años o más en cada lugar y los que no, en lo que respecta a auto percepción sobre sentido de orientación (ANOVA (F: 6.0, Sign 0.16, p>0.05).

\section{Tiempos de respuesta}

El tiempo de respuesta promedio por pregunta fue de 4.7 segundos $(\mathrm{DE}=$ $2.7^{\prime}$ ), con variaciones menores entre los ascensores Polanco (promedio 4.4, DE = 2.5), y Espíritu Santo (promedio 5, DE = 1.7), tal como muestra la tabla 2.

No se encontraron diferencias significativas entre hombres y mujeres en lo relativo a tiempos de respuesta $(\mathrm{t}(133)=1.2, \mathrm{p}=.022)$, no obstante lo cual las mujeres demoraron, en promedio, casi medio segundo más por estimación. Tampoco se detectó una asociación entre la edad de las personas y el tiempo que demoraron en responder el test. Esto fue revelado tanto por una correlación simple entre las variables (Pearson=0.049), como por un análisis de varianza (ANOVA F: 2.43 p> 0.05 ), donde la población se dividió en los tres grupos mencionados anteriormente. Por último, no se encontraron diferencias en el tiempo promedio por estimación tomado por las personas en las plataformas superiores e inferiores $(\mathrm{t}(73)=3.44$, $\mathrm{p}=.01$ ), a pesar de que los que respondieron en las plataformas superiores se demoraron un total de 28.3 segundos en responder a todas las preguntas $(\mathrm{SD}=11.7)$, mientras los que lo hicieron abajo se tomaron 19.1 segundos $(\mathrm{SD}=10.8)$.

Sin embargo, se encontraron diferencias estadísticamente significativas entre el nivel de auto-percepción sobre sentido de orientación y el tiempo de respuesta. Un análisis de varianza (ANOVA) mostró que aquellos que se consideraron a sí mismos con buen sentido de orientación demoraron, en promedio, 4.57' por estimación, mientras los que se consideraron con regular o mal sentido de orientación demoraron 4.11' y 6.8 segundos respectivamente (ANOVA F: 6.73 p> 0.05). 


\section{Error Promedio por Persona}

El cálculo de EPP se realizó calculando el diferencial entre lo señalado por las personas con la aguja giratoria, y la línea virtual entre éstas y cada uno de los cinco hitos. Para este cálculo se consideró que esta línea virtual llegaba al centro del hito. Lo anterior permitió tener un valor de error para cada estimación. Al sumarse estos valores y dividirse por el total de estimaciones (5), se obtuvo un valor promedio de error por estimación.

El análisis de estos resultados detectó que no existieron diferencias estadísticamente significativas en el EPP entre hombres y mujeres $(\mathrm{t}(133)=.916, \mathrm{p}=$ .0362 ), aunque las mujeres fueron, en promedio, más precisas que los hombres en alrededor de $2^{\circ}$. Asimismo, no se detectaron diferencias entre el EPP y la edad (ANOVA, F: 2.43 p> 0.05), ni entre la auto-percepción sobre la capacidad de orientarse y el error promedio (ver tabla 2).

El tiempo de residencia tampoco estuvo asociado al EPP. Un análisis de varianza mostró que aquellos que habían vivido en el cerro Polanco o en el Bellavista por más de cinco años tuvieron un error promedio por estimación similar a aquellos que habían vivido menos de cinco años (F: .794 Sig .374, p> 0.05). Sin embargo, sí se detectaron diferencias significativas en el error promedio entre aquellos que hicieron sus estimaciones en las plataformas superiores o inferiores. En efecto, el EPP de aquellos que hicieron sus estimaciones en las plataformas superiores fue de 29.04 grados, mientras que este valor fue de solo 19.04 grados en las plataformas inferiores $(\mathrm{t}(133)=4.79,(\mathrm{~F}: 7.26) \mathrm{p}=.000$. Este fenómeno ocurrió con mayor intensidad en el ascensor Espíritu Santo, donde el error promedio en la plataforma superior fue de 36.9 grados y en la inferior 22.4 grados, que en el ascensor Polanco, donde estas diferencias fueron de 21.9 y 17.8 grados respectivamente.

\section{5.- Discusión}

Varios son los aspectos de interés de los resultados anteriores. Primero, el hecho que la auto-percepción sobre el sentido de orientación no esté relacionada con el error promedio de las estimaciones hechas por las personas, si bien es un hecho llamativo, tal como lo muestran varios estudios en la materia (STREETER; VITELLO, 1986; MALINOWSKY; GILLESPIE, 2001), no es tampoco infrecuente. En efecto, estudios realizados por campus universitarios (KOZLOWSKI; BRYANT, 1977) o en hospitales (MOESER, 1988), ha mostrado que la auto-evaluación sobre el sentido de evaluación no está necesariamente relacionada con una buena orientación. Lo mismo puede decirse de la relación familiaridad-error en la estimación, como lo mostró un estudio realizado en un campus universitario (KIRASIC; ALLEN; SIEGEL, 1984).

Sin embargo, el resultado más sorprendente e intrigante de este experimento es el hecho de que las estimaciones resultaron más precisas en las plataformas inferiores que en las superiores (contradiciendo de este modo la hipótesis inicial de este estudio). Esto parece contrario a lo señalado en la mayoría de los estudios 
de cognición espacial, que sugieren que la formación de un marco de referencia general que encapsule la ubicación de lugares e hitos del medio ambiente, es esencial para que las personas se orientarse eficientemente.

Una posible explicación para lo anterior es que la existencia de ciertos hitos en el paisaje que condicionarían las estimaciones. La evidencia al respecto indica que las estimaciones hechas por las personas se ven afectadas tanto por la dirección de la red de calles donde se hace la estimación (los individuos alinearían sus estimaciones a esta red de calles, MONTELLO, 1991), como por el interés que ciertos hitos despiertan en las personas (SMITH, 1984). Otra posible explicación es que las personas hayan usado estrategias diferentes al momento de realizar las estimaciones en las plataformas superiores e inferiores. De acuerdo a esta hipótesis, al momento de realizar las estimaciones superiores las personas habrían usado, en vista de la relativa lejanía de esta estimación de una "acción concreta" (en el sentido de tener que efectivamente tener que dirigirse al lugar señalado, una racionalidad categórica, con el objeto de reducir la demanda cognitiva de la tarea (WORBOYS; DUCKHAM; KULIK, 2004) y así facilitar su memorización. Al respecto, existe evidencia de que las personas memorizan información espacial con distintos grados de "granularidad" (es decir, menos precisar y completas), de acuerdo a la escala de aproximación a la realidad. Tomko (TOMKO; WINTER, 2006), por ejemplo, mostró que las personas, al momento de dar instrucciones verbales sobre cómo llegar a un destino a un tercero, se hacen más detalladas y precisas (aumentan su granularidad) en la medida que se acercan a sus destinos. Esto respondería a la necesidad de manejar mentalmente grandes cantidades de información del medio ambiente (HERNÁNDEZ, 1994), generando la disparidad observada entre las evaluaciones en las plataformas superiores e inferiores. Al contrario, en la medida que las personas se encontraban más cercanas a la toma de decisiones efectivas en las plataformas inferiores, el nivel de atención ante la tarea pudo haber sido mayor. Futuras investigaciones en esta línea deberían ahondar en estas líneas. 


\section{REFERENCIAS}

ALLEN, G.; SIEGEL, A.; ROSINSKI, R. The role of context in structuring spatial knowledge. Journal of Experimental Psychology: human learning and memory, v. 4, n. 6, p. 617-630, 1978.

APPLEYARD, D. Styles and methods for structuring a city. Environment and Behavior Journal, n. 2, p. 100-117, 1970.

CARLSON, L. A. et al. Getting lost in buildings. Current Directions in Psychological Science, Atlanta, v. 19, n. 5, p. 284-289, 2010.

COLUCCIA, E.; MARTELLO, A. Il ruolo della memoria di lavoro visuo-spaziale nell'orientamento geografico: uno studio correlazionale (The role of VSWM in geographical orientation: a correlational study). Giornale Italiano di Psicologia, n. 3, p. 523-552, sett. 2004.

DENIS, M. F. et al. Spatial discourse and navigation: an analysis of route directions in the city of Venice. Applied Cognitive Psychology, n. 13, p. 145-174, 1999.

DOWNS, R.; STEA, D. Cognitive maps and spatial behavior: image and environment. London: Edward Arnold, 1973.

FOLEY, J.; COHEN, A. Mental mapping of a megastructure. Canadian Journal of Psychology, n. 38, p. 440-453, 1984.

GÄRLING, T.; LINDBERG, E.; MANTYLA, T. Orientation in buildings: effects of familiarity, visual access and orientation aids. Journal of Applied Psychology, v. 68, n. 1, p. $177-186,1983$.

GÄRLING, T. et al. Cognitive mapping of large-scale environments: the interrelation of action plans, acquisition and orientation. Environment and Behavior Journal, n. 16, p. 3-30, 1984.

GOLLEDGE, R. G. Place recognition and wayfinding: making sense of space. Geoforum, v. 23, n. 2, p. 199-214. 1992.

GOLLEDGE, R. G. Path selection and human preference in human navigation: a progress report. In: CONFERENCE ON SPATIAL INFORMATION THEORY - COSIT, 2,. 1995, Semmering, Austria. Proceedings... Semmering: Springer (Lecture Notes in Computer Science), 1995, n. 988, p. 207-222.

GOLLEDGE, R. G.; STIMSON, R. J. Spatial Behavior: a geographical perspective. New York: Guilford, 1997. 
GOLLEDGE, R. G. et al. A conceptual model and empirical analysis of children's acquisition of spatial knowledge. Journal of Environmental Psychology, n. 5, p. 125-152, 1985.

GOLLEDGE, R. G. et al. Integrating route knowledge in an unfamiliar neighborhood: along and across route experiments. Journal of Environmental Psychology, n. 13, p. 293-307, 1993.

HERNÁNDEZ, D. Qualitative representation of spatial knowledge. Berlin: Springer, 1994. Series: Lecture Notes in Artificial Intelligence 804.

HOLDING, C. S.; HOLDING, D. H. Acquisition of route network knowledge by males and females. The Journal of General Psychology, v. 116, n. 1, p. 29-41, 1989.

HÖLSHER, C. et al. Up the Down Staircase: Wayfinding Strategies and MultiLevel Buildings. Journal of Environmental Psychology, v. 26, n. 4, p. 284-299, 2006.

KIRASIC, K.; ALLEN, G.; SIEGEL, A. Expression of configurational knowledge of large-scale environments: students' performance of cognitive tasks. Environment and Behavior Journal, v. 16, n. 6, p. 687-712, 1984.

KIRASIC, K. Age differences in adults' spatial abilities, learning environmental layout, and wayfinding behavior. Spatial Cognition and Computation, n. 2, p. 117-134, 2000.

KITCHIN, R. Are there sex differences in geographic knowledge and understanding? The Cartographic Journal, v. 163, n. 3, p. 273-286, 1996.

KLIPPEL, A.; MONTELLO, D. R. Linguistic and nonlinguistic turn directions concepts. In: INTERNATIONAL CONFERENCEON SPATIALINFORMATION THEORY - COSIT, 8., 2007, Melbourne, Australia. Proceedings... Melbourne: Springer (Lecture Notes in Computer Science), 2007, n. 4736, p. 354-372.

KOZLOWSKI, L.; BRYANT, K. Sense of direction, spatial orientation and cognitive maps. Journal of Experimental Psychology: human perception and performance, v. 3, n. 4, p. 590-598, 1977.

LAWTON, C. A. Strategies for indoor way-finding: the role of orientation. Journal of Environmental Psychology, n. 16, p. 137-145, 1996.

MALINOWSKI, J.; GILLESPIE, W. Individual differences in performance on a large-scale, real-world wayfinding task. Journal of Environmental Psychology, n. 21, p. 3-82, 2001. 
MOESER, S. D. Cognitive mapping in a complex building. Environment and Behavior Journal, v. 20, n. 1, p. 21-49, 1988.

MOFFAT, S. D.; HAMPSON, E.; HATZIPANTELIS, M. Navigation in a virtual maze: sex differences and correlation with psychometric measures of spatial ability in humans. Evolution and Human Behavior, n. 19, p. 73-87, 1998.

MONTELLO, D. Spatial orientation and the angularity of urban routes: a field study. Environment and Behavior Journal, v. 23, n. 1, p. 47-69, 1991.

MONTELLO, D. R. A new framework for understanding the acquisition of spatial knowledge in large-scale environments. In: EGENHOFER, M.; REGINALD, G. (Ed.). Spatial and temporal reasoning in cartographic information systems. Oxford: Oxford University Press, 1992. p. 143-154.

MONTELLO, D. R.; PICK, H. L. Integrating knowledge of vertically aligned large-scale spaces. Environment and Behavior Journal, n. 25, p. 457-484, 1993.

MONTELLO, D. R. et al. Sex-related differences and similarities in Environmental and Gepgraphic spatial abilities. Annals of the Association of American Geographers, v. 89, n. 3, p. 515-534, 1999.

O'LAUGHLIN, E. M.; BRUBAKER, B. S. Use of landmarks in cognitive mapping: gender differences in self report versus performance. Personality and Individual Differences, v. 24, n. 5, p. 595-601, 1998.

SAUCIER, D. M. et al. Are sex differences in navigation caused by sexually dimorphic strategies or by differences in the ability to use the strategies? Behavioral Neuroscience, v. 116, n. 3, p. 403-410, 2002.

SCHMITZ, S. Gender related strategies in environmental development: effect of anxiety on wayfinding in and representation of a three-dimensional maze. Journal of Environmental Psychology, n. 17, p. 215-228, 1997.

SIEGEL, A. W.; WHITE, S. H. The development of spatial representations of large-scale environments. In: REESE, H. (Ed.). Advances in Child Development and Behavior. New York: Academic Press, 1975. v. 10, p. 9-55.

SMITH, C. D. The relationship between the pleasingness of landmarks and the judgement of distance in cognitive maps. Journal of Environmental Psychology, n. 4, p. 229-234, 1984.

STECK, S.; MALLOT, H. The role of local and global landmarks in virtual environment navigation. Presence, v. 9, n. 1, p. 69-83, 2000. 
STREETER, L.; VITELLO, M. A profile of drivers' map-reading abilities. Human Factors, v. 28, n. 2, p. 223-239, 1986.

THORNDYKE, P.; HAYES-ROTH, B. Differences in spatial knowledge acquired from maps and navigation. Cognitive Psychology, n. 14, p. 560-589, 1982.

TOLMAN, E. C. Cognitive maps in rats and men. Psychological Review, v. 55, n. 4, p. 189-208, 1948.

TOMKO, M.; WINTER, S. Recursive Construction of Granular Route Directions. Journal of Spatial Science, v. 51, n. 1, p. 101-115, 2006.

TVERSKY, B.; BARATZ, D. Memory for faces: are caricatures better than photographs?. Memory and cognition, v. 13, n. 1, p. 45-49, 1985.

TVERSKY, B. Distortions in memory for maps. Cognitive Psychology, n. 13, p. 407-433, 1982.

TVERSKY, B. Structures of mental spaces: how people think about space. Environmental and Behavior Journal, v. 35, n. 1, p. 66-80, 2003.

WALLER, D.; BEALL, A.; LOOMIS, J. Using virtual environments to assess directional knowledge. Journal of Environmental Psychology, n. 24, p. 105-116, 2004.

WARD, S. L.; NEWCOMBE, N.; OVERTON, W. F. Turn left at the church, or three miles North: a study of direction giving and sex differences. Environment and Behavior Journal, v. 18, n. 2, p. 192-213, 1986.

WORBOYS, M.; DUCKHAM, M.; KULIK L. Commonsense notions of proximity and direction in environmental space. Spatial Cognition and Interaction, v.4, n. 4, p. 285-312, 2004.

Recebido em: 19 de agosto de 2013 Aceito em: 29 de dezembro de 2013 